\title{
参与还原型 $\mathrm{H}^{+}-\mathrm{ATP}$ 酶光活化的质子梯度*
}

\author{
应文龙 即青王健 李波侵
}

(中国科学院上海植物生理研究所)

\section{关策诃 还原型 $\mathrm{H}^{+}-\mathrm{ATP}$ 酶、光活化、区域化和非区域化质子梯度}

水的氧化和还原质醌 (reduced plastoquinone, $\mathrm{PQH}_{2}$ ) 的氧化所释放的质子（proton。 $\mathrm{H}^{+}$) 用于膜上腺苷三磷酸 (adenosine triphosphate, ATP) 酶复合体催化的 ATP 形成. 而 质子从产生部位到 ATP 酶复合体膜内质子通路 (proton channel, $\mathrm{CF}_{0}$ ) 的传导途径还没有 定论. Mitchell 曾提出化学渗透假说 ${ }^{(2)}$, 认为膜内外水相质子浓度差或非区域化质子梯度偶 联 ATP 合成酶. 而Williams 认为局限于膜内或膜内表面的质子流偶联于 ATP 合成烈. 早 期的大量实验结果支持和发展了 Mitchell 假说. 但近来有不少实验室观察到的现象, 难以用 化学渗透假说解释 ${ }^{(3)}$, 所以目前有关区域化和非区域化偶联机制还有争议.

ATP 酶复合体除能催化 ATP 合成反应外, 还能催化 ATP 水解反应, 所以它也被称为 $\mathrm{H}^{+}$-ATP 水解酶 ( $\mathrm{H}^{+}$-adenosine triphosphase, $\mathrm{H}^{+}$-ATPase). 但它具有隐性 ATP 酶性质, 需经二硫苏木幖 (dithiothreitol, DTT) 及光活化, 才有水解 ATP 的活力. 这一光活化过程 包括二硫键还原和酶构象变化. 二硫键的还原可能不需要膜的能化, 因为类妻体膜与 DTT 暗 中保温 $2 \mathrm{~h}$ 就可导致酶被疏基修饰. 因此, 预先将 $\mathrm{H}^{+}-\mathrm{ATP}$ 酶处理为还原型, 则光的活化只与 酶的构象变化有关,这一构象变化是被光合电子传递产生的质子梯度所推动.

McCarty 等研究了叶绿体光下电子传递、质子吸收、ATP 合成三者之间的关系, 结果与化 学渗透假说相符合 ${ }^{[4,5]}$. Gräber 等建立了暗中还原型 $\mathrm{H}^{+}-\mathrm{ATP}$ 酶活化对酸碱转换产生的质 子差的依赖关系 ${ }^{[6]}$. 本文研究了光下还原型 $\mathrm{H}^{+}-\mathrm{ATP}$ 酶活化与光合电子传递和光诱质子差 $(\Delta \mathrm{pH})$ 间的关系, 辩证质子从产生部位到 ATP 酶复合体膜内质子通路 $\left(\mathrm{CF}_{0}\right)$ 的可能传导途 径.

\section{一、材料和方法}

1. 村料 大田菠菜 (Spinacia oleracea L.) 叶.

2. 制各 疏基修饰类衰体膜: 将常规制备的叶绿体在含 $2 \mathrm{mmol} / \mathrm{L} \mathrm{Mg} \mathrm{Cl} 、 10 \mathrm{mmol} / \mathrm{L}$ $\mathrm{NaCl} 、 0.15 \mathrm{~mol} / \mathrm{L}$ 葡萄糖溶液中洗二次, 在含 $15 \mathrm{mmol} / \mathrm{L} \mathrm{DTT} 、 5 \mathrm{mmol} / \mathrm{LMg} \mathrm{Cl}_{2} 、 25 \mathrm{mmol} / \mathrm{L}$. $\mathrm{K} \mathrm{Cl} 、 15 \mu \mathrm{mol} / \mathrm{L}$ 甲基硫酸葱（phenazine methosalfate, PMS) $、 0.1 \mathrm{~mol} / \mathrm{L}$ 葡萄糖、 $0.2 \mathrm{mg} / \mathrm{ml}$ 叶绿素 (chlorophyll, Chl) 的溶液中照光 $5 \mathrm{~min}$, 转暗后保温 $10 \mathrm{~min} 于 15^{\circ} \mathrm{C}$, 即为实验所需 的疏基修饰类豪体膜.

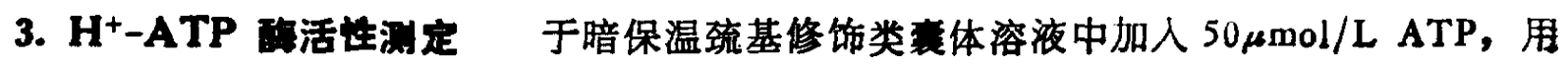

本文1988年12月31 日收到. 1985 年 4 月 25 日收到改稆。

* 国家自然科学著全完助项目. 
机械块门控制闪光活化 $\mathrm{H}^{+}-\mathrm{ATP}$ 酶。加稀释液稀释 4 倍, 稀释液含 $25 \mathrm{mmol} / \mathrm{L} \mathrm{Tris}-\mathrm{HCl} \mathrm{pH}$

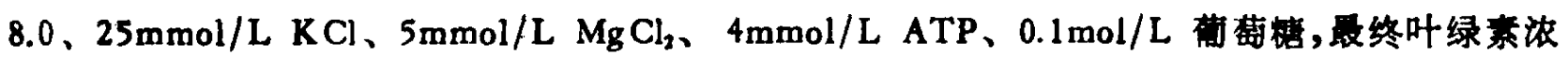
度为 $0.05 \mathrm{mg} / \mathrm{L}$. 此时将反应管迅速从 $15^{\circ} \mathrm{C}$ 转到 $37^{\circ} \mathrm{C}$, 保温 $2 \mathrm{~min}$, 加 $20 \%$ 三氯乙酸停止反 应. ATP 水解所释放的无机磷按文献[7]报道测定.

4. 叶绿体 9-基基叫啶 (9-aminoacridin, 9-AA) 苂光测定 疏基修饰类莫体膜加人反 应液含 $10^{-5} \mathrm{~mol} / \mathrm{L} 9-\mathrm{AA} 、 2 \mathrm{mmol} / \mathrm{L}$ DTT、 $5 \mathrm{mmol} / \mathrm{L} \mathrm{MgCl}_{2} 、 25 \mathrm{mmol} / \mathrm{L} \mathrm{KCl} 、 0.15 \mathrm{~mol} / \mathrm{L}$ 荡萄糖、 $40 \mathrm{nmol} / \mathrm{L}$ 绕氨毒素及 $100 \mu \mathrm{mol} / \mathrm{L}$ 甲基紫精, 使最终 $\mathrm{Chl}$ 浓度为 $10 \mu \mathrm{g} / \mathrm{ml}$. 并根据 要要加或不加 $0.2 \mathrm{mmol} / \mathrm{L}$ ATP.

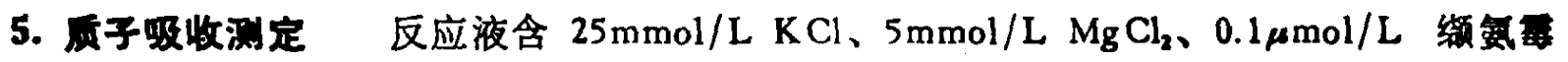
系、 $10 \mu \mathrm{mol} / \mathrm{L}$ PMS、0.15 mol $/ \mathrm{L}$ 葡萄糖. 详见文献 [8].

叶绿素含是测定见文献 [8].

\section{二、结 果}

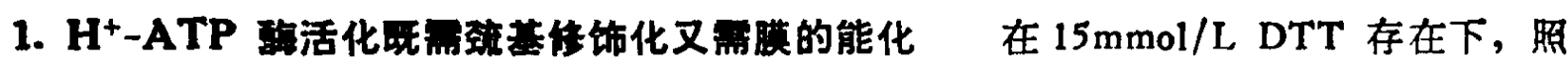
光 $5 \mathrm{~min}$ 后类莀体 ATP 酶活力达到最大, 转暗后, 活力迅速衰减到对照（不加 DTT) 水平, 约 需 3min。此时若给予 $2 \mathrm{~s}$ 照光, 就可以完全恢复 $\mathrm{H}^{+}-\mathrm{ATP}$ 酶的最大活性 (图 1). 第一次照光 $\mathrm{H}^{+}-\mathrm{ATP}$ 酶除能化构变外,还有二硫键的还原反应. 而第二次照光仅与偶联因子 $\left(\mathrm{CF}_{2}\right)$ 的构 变有关。显然类毫体 $\mathrm{H}^{+}-\mathrm{ATP}$ 酶活化既需二硫键还原, 又需膜能化。而还原型 $\mathrm{H}^{+}-\mathrm{ATP}$ 酶 活化仅需膜能化. 我们利用还原型 $\mathrm{H}^{+}-\mathrm{ATP}$ 酶研究了光下质子吸收与 $\mathrm{H}^{+}-\mathrm{ATP}$ 酶活化的关 系。

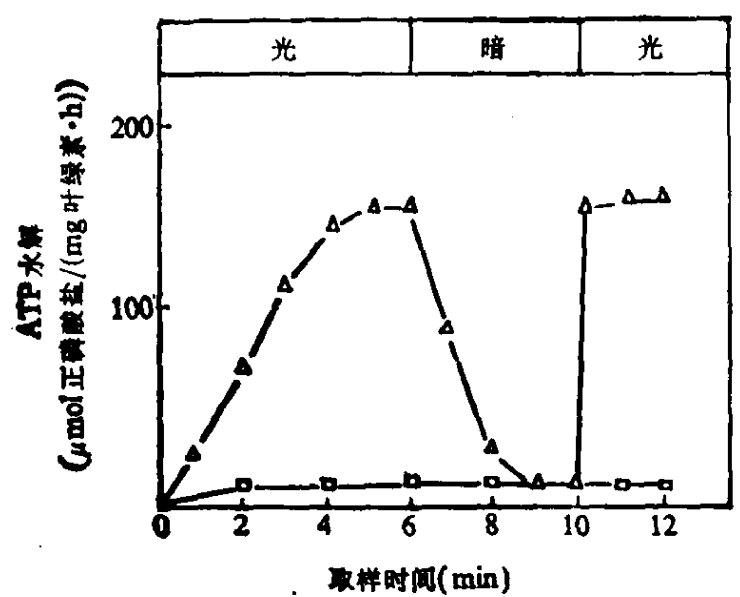

1 1 DTT 存在下类毫体膜 $\mathrm{H}^{+}-$ATP 酶的活化 a—口 对用; $\Delta-\Delta$ 加 $15 \mathrm{mmol} / \mathrm{L}$ DTT

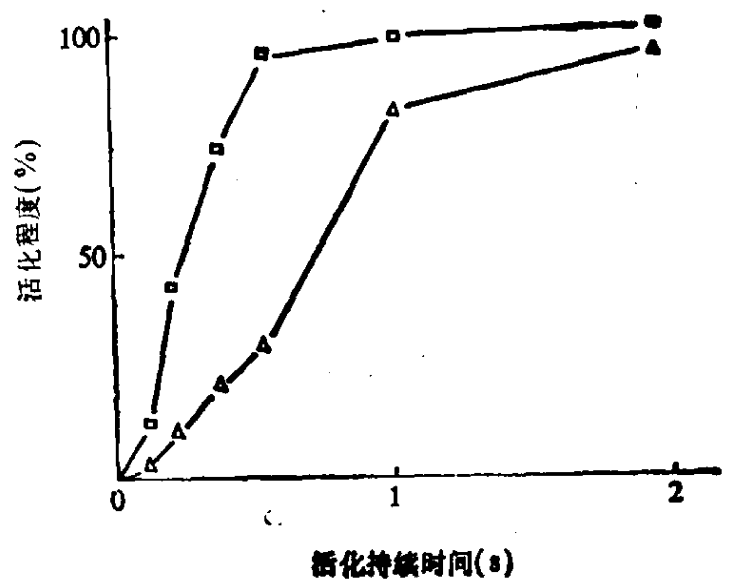

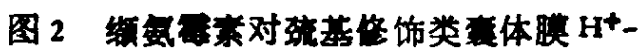
ATP 酶光佸化的影响

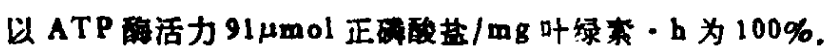

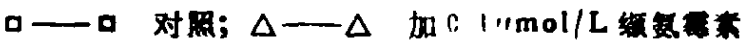

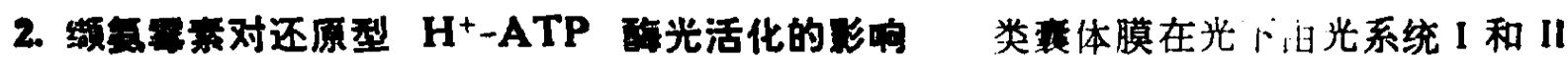
反应中心做发电子, 推动电子递体的氧化还原, 反应过程中由质子泵向膜内转导质子, 形成跨 瞙质子动力势 $\left(\Delta \bar{\mu}_{\mathrm{H}} \cdot\right)$ ，它包括跨膜电位差 $(\Delta \boldsymbol{\phi})$ 和跨膜质子差 $(\Delta \mathrm{pH}) . \Delta \psi$ 可以利用适当

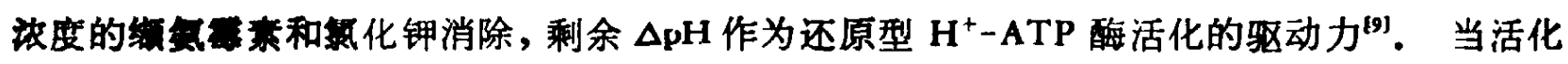
介质中存在 $0.1 \mathrm{mmol} / \mathrm{L}$ 浣氨每意时, 还原型 $\mathrm{H}^{+}-\mathrm{ATP}$ 酶达到最大活力的照光时间需延长 4 倍 
(图 2), 即从 $0.5 \mathrm{~s}$ 延至 2s.

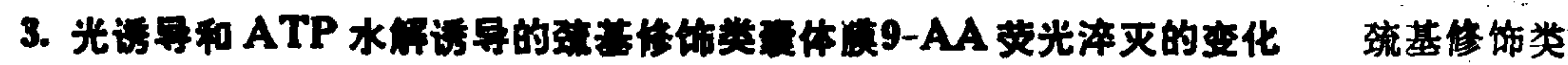

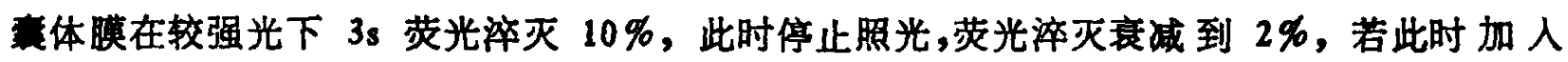
$0.2 \mathrm{~mol} / \mathrm{L} \mathrm{ATP}$, 则茨光淬灭程度继续增加到 $38 \%$. 在低效光强下荻光淬灭达到 $10 \%$ 时需照 光 $10 \mathrm{~s}$, 转暗后则降到 $2 \%$, 若有 ATP 存在,荧光淬灭从 $10 \%$ 下降到 $8 \%$ (见表 1).

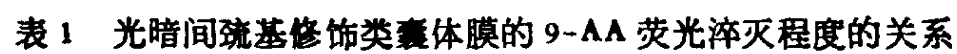

\begin{tabular}{|c|c|c|c|c|}
\hline & & \multicolumn{2}{|c|}{ 光 中 } & \multirow{2}{*}{$\frac{\text { 暗中 }}{\text { 荧光泍灭程度(\%) }}$} \\
\hline & & 盟光时间 (s) & 荧光淬灭程度 (\%) & \\
\hline \multirow{2}{*}{ 盖 光下 } & 天 $\boldsymbol{A T P}$ & 3 & 10 & 2 \\
\hline & 有 ATP & 3 & 10 & 38 \\
\hline \multirow{2}{*}{ 酒光下 } & 无 A「P & 10 & 10 & 2 \\
\hline & 有 ATP & 10 & 10 & 8 \\
\hline \multirow{2}{*}{ 强光下加 $1 \mu \mathrm{mol} / \mathrm{L} \mathrm{DCMU}$} & 无 $\mathbf{A T P}$ & 11 & 10 & 2 \\
\hline & 有 ATP & 11 & 10 & 8 \\
\hline
\end{tabular}

生: 以 10-'mol/L 9-AA 荻光为 $100 \%$.

9- $\mathrm{AA}$ 荧光淬灭程度反映了推动 $\mathrm{H}^{+}-\mathrm{ATP}$ 酶活化的 $\Delta \mathrm{pH}$ 大小，而暗中有 ATP 存在时的

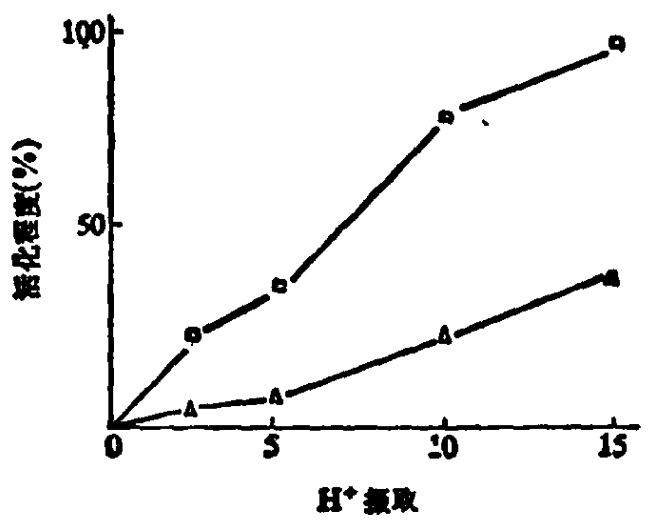

因 $3 \mathrm{H}^{+}-\mathrm{ATP}$ 酶光活化程度与 $\mathrm{H}^{+}$振取的关系

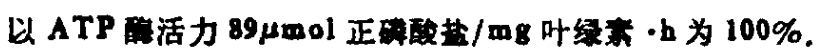

口—口高光强; $\Delta$ 一 $\Delta$ 低光强 苂光淬灭程度则表示 $\mathrm{H}^{+}-\mathrm{ATP}$ 酶质子㬌活 性高低。所以比较光下和暗中荻光淬灭程度， 可以找出 $\Delta \mathrm{pH}$ 与 $\mathrm{H}^{+}-\mathrm{ATP}$ 酶活化之间的关

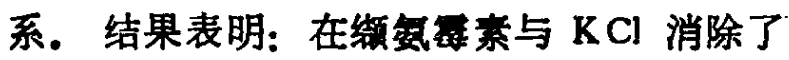
$\Delta \boldsymbol{\psi}$ 条件下, 中调节照光时间保持 $\Delta \mathrm{pH}$ 恒定， $\mathrm{H}^{+}$-ATP 酶活化程度仍随照光强度的减弱而 下降。

表 1 中还表明，二氮酚二种豚 (diphenoldimethylurea，DCMU）抑制了光合电子 传递速度时，虽然 9-AA 荻光淬灭程度一致， $\mathrm{H}^{+}$-ATP 酶活化程度仍下降(表 1)。

4. 还原型 $\mathrm{H}^{+}-\mathrm{ATP}$ 政光活化与质子吸 收问的可致关而光合电子传递引起质子由介质向腔内转导, 结果使介质相 $\mathrm{pH}$ 上升. 疏

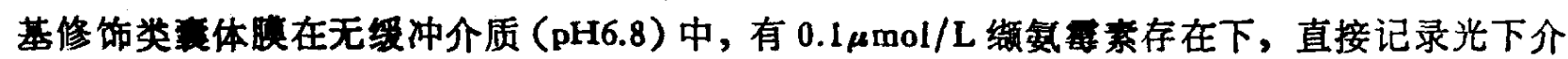
质 $\mathrm{pH}$ 变化, 可以计出 $\mathrm{H}^{+}$吸收量, 以它为横坐标, 在同样条件下检测 $\mathrm{H}^{+}-\mathrm{ATP}$ 酶活化的时 间过程为纵坐标作国(图 3).表明 $\mathrm{H}^{+}-\mathrm{ATP}$ 酶活化程度与质子吸收的函数关系受光强的影响， 即受光合电子传递速度的影响。

\section{三、讨 论}

按炤 Mitchell 的化学透偶联假说 ${ }^{[\mathrm{n}}$,类衰体膜 $\mathrm{H}^{+}-\mathrm{ATP}$ 酶光活化间接受限于光合电子

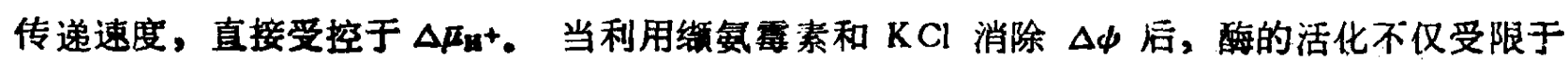


$\Delta p H ， 也$ 受限于光合电子传递速度 (表 1、图 3).

按照 Williams 区域化质子梯度偶联假说，氛化还质子㬌是通过膜上或膜内表面区域 化质子梯度偶联于 $\mathrm{H}^{+}-\mathrm{ATP}$ 酶的光活化. 无论那种假说 $\mathrm{H}^{+}-\mathrm{ATP}$ 酶活化都受控于光合电子 传递速度。而区域化质子梯度的建立要比非区域化质子梯度的建立快得多 ${ }^{[00]}$. 当光合电子传 递速度不同时(光强不同), 通过调节照光时间, 非区域化质子梯度一致的条件下, 区域化质子 梯度则取决于氧化还原质子泵的运转，从而导致 $\mathrm{H}^{+}-\mathrm{ATP}$ 酶受活化程度不同。

实验证明，膜上区域化质子梯度也参与了琉基修饰类荃体膜的光活化过程. 也就是说，光 合氧化还原所释放的质子既可以从腔内介质相,也可以从膜相传导到 $\mathrm{CF}_{0}-\mathrm{CF}_{1}$ 复合体。

[1] Mitchell, P., Nature (London), 191(1961), 144-148.

[2] Williams, R. S. P., J. Theor. Biol., 1 (1961), 1-17.

[3] Dilley, R. A., Ann. Rev. Plant Physiol., 38(1987), 347-418.

[4] Davenport, J. W. and McCarty, R. E., Biochim. Biophys. Acra, 589(1980), 353-357.

[5] Davenport, J. W. and McCarty, R. E., Biochim. Biophys. Acre, 768(1984), 363-374.

[6] Graber, P. et al., in Progress in Photosymuhesis Research. (ed. Biggint, J.), Providence, III (1986), $173-176$.

[7] Ying, W. L. and Li, S. J., Acta Biochim. Biophys. Sinica, 21(1989), 151-158.

[8] Li, S. J.et al., Scientia Sinica, Ser. B, 27(1984), 1147-1161.

[9] Ort, D. R. and Dilley, R. A., Biochim. Biophys. Acre, $49(1976), 108-124$

[10] William8, R. J. P., FEBS Lett., 85(1978), 9-19. 